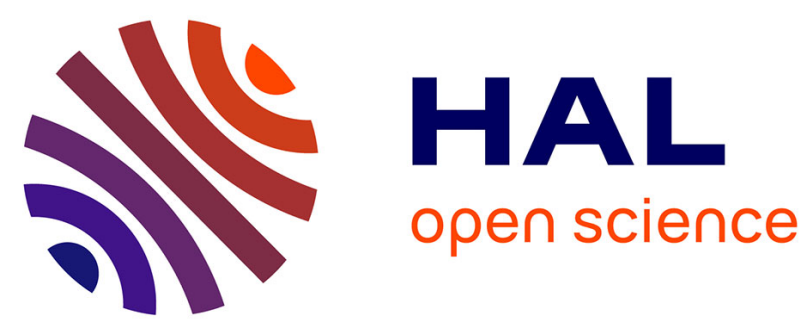

\title{
Relevance of weak and strong classical scattering for the giant negative magnetoresistance in two-dimensional electron gases
}

B Horn-Cosfeld, J Schluck, J Lammert, M Cerchez, T Heinzel, K Pierz, H W

Schumacher, D Mailly

\section{To cite this version:}

B Horn-Cosfeld, J Schluck, J Lammert, M Cerchez, T Heinzel, et al.. Relevance of weak and strong classical scattering for the giant negative magnetoresistance in two-dimensional electron gases. Physical Review B, 2021, 104, 10.1103/physrevb.104.045306 . hal-03376881

\section{HAL Id: hal-03376881 \\ https://hal.science/hal-03376881}

Submitted on 13 Oct 2021

HAL is a multi-disciplinary open access archive for the deposit and dissemination of scientific research documents, whether they are published or not. The documents may come from teaching and research institutions in France or abroad, or from public or private research centers.
L'archive ouverte pluridisciplinaire HAL, est destinée au dépôt et à la diffusion de documents scientifiques de niveau recherche, publiés ou non, émanant des établissements d'enseignement et de recherche français ou étrangers, des laboratoires publics ou privés. 


\title{
Relevance of weak and strong classical scattering for the giant negative magnetoresistance in two-dimensional electron gases
}

\author{
B. Horn-Cosfeld $\odot$ J. Schluck $\odot$, J. Lammert, M. Cerchez, and T. Heinzel $\odot^{*}$ \\ Institut für Experimentelle Physik der kondensierten Materie, Heinrich-Heine-Universität, Universitätsstraße 1, 40225 Düsseldorf, Germany \\ K. Pierz $\odot$ and H. W. Schumacher \\ Physikalisch-Technische Bundesanstalt, Bundesallee 100, 38116 Braunschweig, Germany \\ D. Mailly \\ CNRS, Univ. Paris-Sud, Université Paris-Saclay, C2N Marcoussis, 91460 Marcoussis, France
}

(Received 7 May 2021; revised 7 July 2021; accepted 7 July 2021; published 19 July 2021)

\begin{abstract}
The giant negative magnetoresistance (GNMR), observed in two-dimensional electron gases of high mobility, is studied by controlled definition of additional short-range scatterers in form of two-dimensional Lorentz arrays of varying obstacle density, as well as in the presence of edge scattering. The results support models, which ascribe the temperature-independent regime of the GNMR to strong, classical scattering and the temperaturedependent regime to electron-electron interactions under the influence of mixed disorder. The threshold magnetic field, which separates the two regimes, is in rough agreement with the lower percolation transition of the Lorentz array. At large obstacle densities, interaction corrections are suppressed and memory effects become more relevant. Shape, amplitude, and width of the GNMR depend sensitively on the time scales of the contributing scattering mechanisms. This can lead to qualitatively similar shapes for quite different parameter values.
\end{abstract}

DOI: 10.1103/PhysRevB.104.045306

\section{INTRODUCTION}

Recent transport experiments on two-dimensional electron gases (2DEGs) of high mobility in $\mathrm{Ga}[\mathrm{Al}] \mathrm{As}$ heterostructures have shown the giant negative magnetoresistance (GNMR) [1-9]. Often [3-7] but not always [6-9] the GNMR structure is composed of a narrow, approximately parabolic, peak with its center at $B=0$ on top of a broader structure, which has a maximum at $B=0$, and a minimum at intermediate magnetic fields around $100 \mathrm{mT}$. The narrow peak is quite robust with respect to thermal activation and additional in-plane magnetic fields. This phenomenology is different than that one of similarly textured peaks observed in samples of lower mobilities and larger interaction parameters [10,11], where the narrow peak is strongly temperature dependent and can be explained by weak localization.

The narrow peak of the GNMR texture has been attributed to memory effects by strong, elastic scattering at oval defects, which explains its insensitivity to in-plane magnetic fields as well as to elevated temperatures [5]. In other experiments, a narrow peak centered at $B=0$ or at nonzero magnetic fields has been observed but has remained undiscussed [6,7]. The second structural element of the GNMR, namely the broader peak, which is in most cases (but not all, see Ref. [9]) more sensitive to thermal activation and to in-plane magnetic fields $[3,5,6]$, has been explained by an interplay between mixed disorder and electron-electron interactions [6]. Interactions

\footnotetext{
*Thomas.Heinzel@hhu.de
}

have also been considered within a hydrodynamic description, where the boundary conditions of the viscous electron flow at the edges have a global effect on the conductivity $[12,13]$. Within this description, however, the dependence on $B_{\|}$has remained an open issue. Furthermore, a quantum mechanical treatment has been presented [14], based on earlier observations that $B_{\|}$modifies the scattering rate of electrons in closely spaced Landau levels $[2,15]$. Thus, it has emerged that a variety of effects contribute simultaneously to the GNMR, with weighting factors that depend on the sample parameters. Presently, a comprehensive model for all these contributions is not available. However, it is clear that the disorder potential landscape has a crucial influence on the appearance of the GNMR. Therefore, studying the dependence of the GNMR on designed disorder potentials with varying parameters may contribute to a better understanding of this remarkable effect.

In the present paper, we implement this concept by preparing two-dimensional Lorentz gases (2DLGs) [16], i.e., arrays of identical, circular obstacles at random positions in semiconductor heterostructures, which show the GNMR. The phenomenology of the samples without the designed disorder is very similar to that one reported by Bockhorn et al. [3,5] as well as by Hatke et al. [6]. The particular type of disorder potential was chosen for several reasons. First of all, the arrays can be prepared with excellent control of their scattering parameters. Second, the sparse but strong obstacles mimic some possible intrinsic scenarios, like scattering at oval defects [5] or at sparse impurities. Finally, the analysis and interpretation of the measurements can resort to the well-established transport models for two-dimensional Lorentz gases [17-22]. 
In Sec. II, the sample preparation and the experimental setup is presented. The measured magnetoresistance phenomenology is presented in Sec. III, followed by an interpretation and discussion in Sec. IV. The paper ends with a summary and our conclusions (Sec. V).

\section{PREPARATION OF THE SAMPLES AND EXPERIMENTAL SETUP}

A GaAs $/ \mathrm{Al}_{0.3} \mathrm{Ga}_{0.7} \mathrm{As}$ heterostructure with a $2 \mathrm{DEG}$ $150 \mathrm{~nm}$ below the surface was prepared by molecular beam epitaxy. After a brief illumination with an infrared lightemitting diode, an electron density of $n_{e}=2.5 \times 10^{15} \mathrm{~m}^{-2}$ and a mobility of $\mu=960 \mathrm{~m}^{2} / \mathrm{Vs}$ of the pristine $2 \mathrm{DEG}$ is measured at liquid helium temperatures, corresponding to an electronic mean free path of $\ell_{e}=78 \mu \mathrm{m}$. In order to avoid strain and hysteresis effects, which can decrease the mobility, Schottky-type electrodes on the sample surface are avoided.

The obstacle arrays are patterned by electron beam lithography and subsequent reactive ion etching. Each obstacle corresponds to an etched circle with a geometric radius of $425 \mathrm{~nm}$, see the left inset in Fig. 1(a). Similar arrays with an obstacle radius of $75 \mathrm{~nm}$ have been studied as well and are shown in the supplement [23]. Random distribution is ensured by generating the center coordinates of the circles with a random number generator, and the correct distribution is verified by a Voronoi tesselation of the array and a subsequent check of the distribution function for the Voronoi cell areas [24,25]. Here, we allow mutual overlap of the obstacles, in accordance with the assumptions underlying the widely used theoretical results for the magnetotransport of 2DLGs [18,26-28]. A picture of a typical sample is shown in the right inset of Fig. 1(a). Since the analysis of Aharonov-Bohm oscillations at large magnetic fields indicates a lateral electronic depletion length of $\approx 75 \mathrm{~nm}$ [29], the effective, electronic obstacle radius is $r_{s} \approx 500 \mathrm{~nm}$. Furthermore, magnetoresistivity experiments on antidot lattices prepared from this wafer by the same method have shown that the electrostatic potential at the obstacles is quite steep [22], and can be approximated by hard walls. The dimensionless number density of a 2DLG is defined as $n^{\star} \equiv n_{s} r_{s}^{2}$. It measures the fraction of the sample area covered by the obstacles in the absence of mutual overlap. The corresponding mean free path of the clean 2DLG equals [20] $\ell_{s} \equiv\left(2 n_{s} r_{s}\right)^{-1}$. Clean 2DLGs of this type undergo a percolation transition at a critical magnetic field [28]:

$$
B_{c}\left(n^{\star}\right)=\frac{\hbar}{e} \sqrt{2 \pi n_{e}} \times \frac{1}{r_{s}} \times \frac{\sqrt{n^{\star}}}{\sqrt{0.359}-\sqrt{n^{\star}}},
$$

which is reproduced in Fig. 1(a) for the small obstacle densities considered here. For $B>B_{c}\left(n^{\star}\right)$, the electrons are localized by skipping around obstacles or clusters thereof.

The length of the Hall bar between the voltage probes as well as its width $w$ was $100 \mu \mathrm{m}$. Since the GNMR is known to depend on $w$ [7], the Lorentz arrays up to $n^{\star}=25 \times 10^{-3}$ have also been prepared in Hall bars of $20 \mu \mathrm{m}$ width, with otherwise identical parameters.

The experiments were carried out in a dilution refrigerator with a base temperature of $8 \mathrm{mK}$. The minimum electron temperature is estimated to $\approx 80 \mathrm{mK}$, which can be increased up to $\approx 800 \mathrm{mK}$ without destabilizing the mixture circuit. For
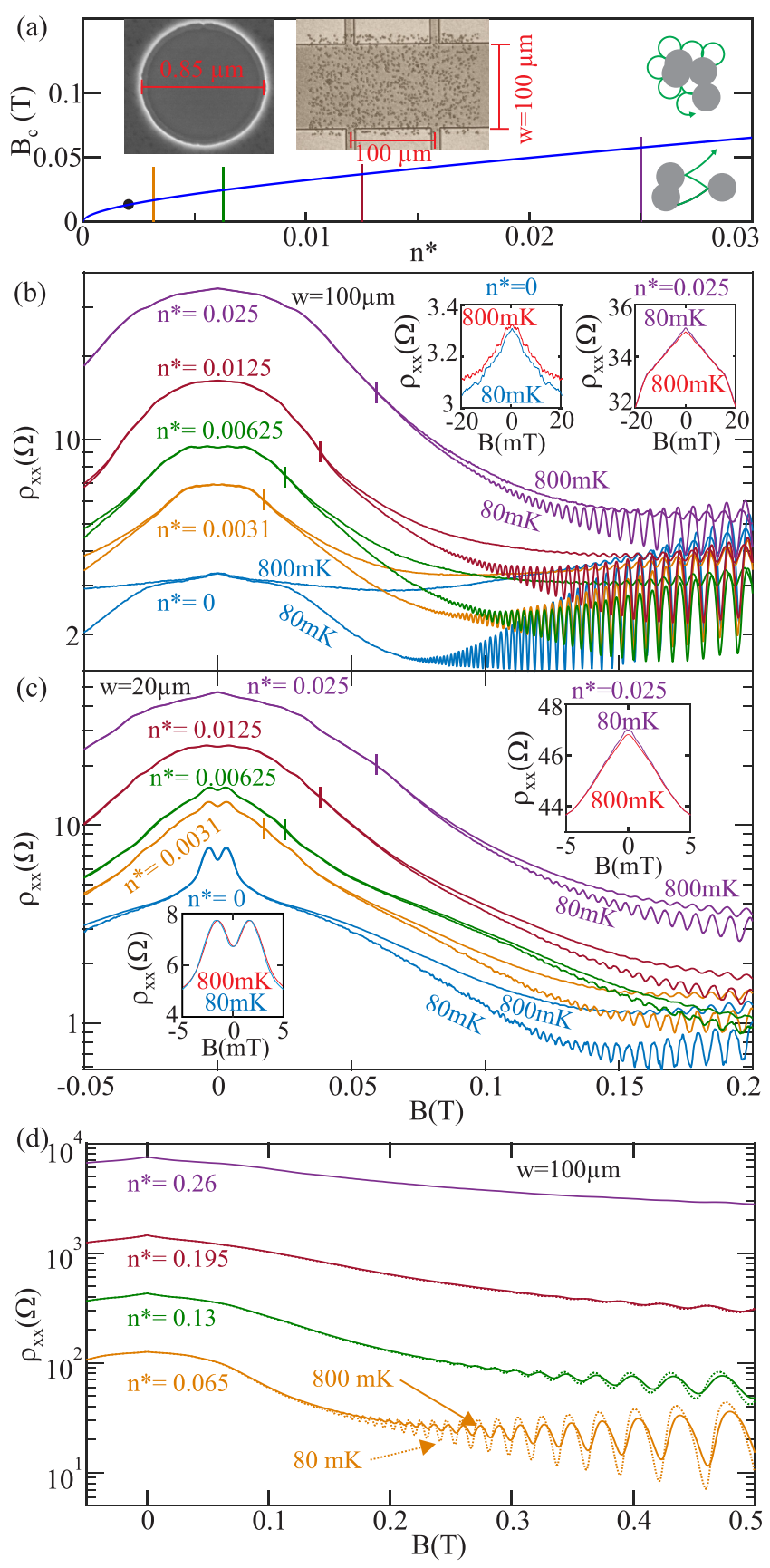

FIG. 1. (a) Scanning electron microscope picture of a single etched circle of $425 \mathrm{~nm}$ nominal radius (left inset) and an optical microscope picture of an exemplifying Lorentz array with dimensionless obstacle density $n^{\star}=1.25 \times 10^{-2}$ (center inset). The main figure shows the lower percolation threshold $B_{c}\left(n^{\star}\right)$ of the 2DLGs (full line). The black circle marks the value estimated for an effective $n^{\star}$ in our pristine heterostructure. Right insets: Example trajectories for the delocalized (bottom) and localized (top) phase. The magnetoresistivities of the arrays for different values of $n^{\star}$ and for two Hall bar widths $w$, in particular small $n^{\star}, w=100 \mu \mathrm{m}$, small $n^{\star}$, $w=20 \mu \mathrm{m}$ and large $n^{\star}, w=100 \mu \mathrm{m}$ are reproduced in (b), (c), and (d), respectively. For each case, the traces measured at base temperature $(\approx 80 \mathrm{mK})$ and at $800 \mathrm{mK}$ are shown. The vertical lines denote the 2DLG percolation thresholds from (a). The insets show zoom-ins of the magnetoresistance close to $B=0$ and its temperature dependence for selected samples. 


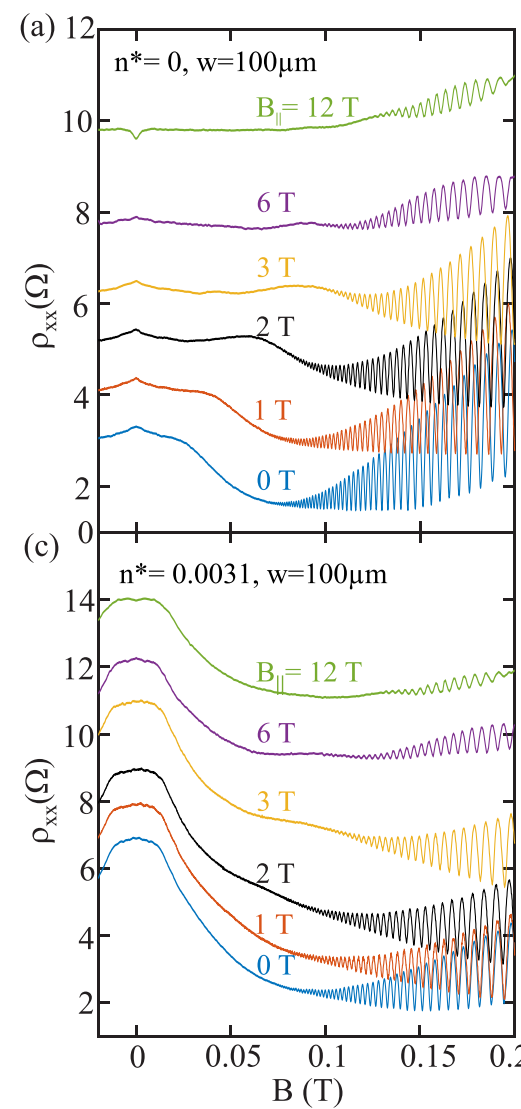

(b)

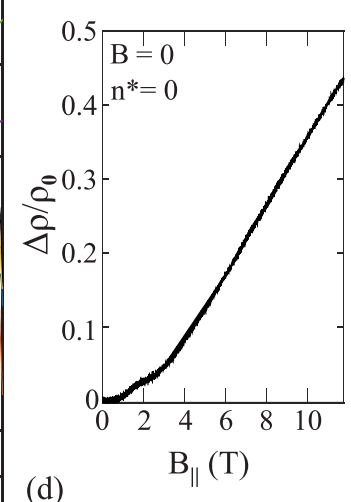

(d)

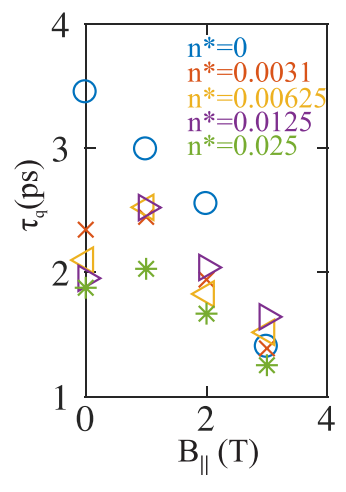

FIG. 2. Dependence of $\rho_{x x}(B)$ on the in-plane magnetic field $B_{\|}$ for the samples of width $w=100 \mu \mathrm{m}$ for $n^{\star}=0$ (a) and 0.0031 (c). Adjacent traces towards increasing $B_{\|}$are offset by $1 \Omega$ for clarity. (b) $\Delta \rho_{x x}\left(B_{\|}\right)$for $B=0$ of the sample with $n^{\star}=0$. The dependence of the quantum scattering time on $B_{\|}$for the samples with $W=100 \mu \mathrm{m}$ is shown in (d). In all measurements shown here, the temperature was $80 \mathrm{mK}$.

larger temperatures, a ${ }^{4} \mathrm{He}$ gas flow cryostat with a variable temperature insert and a base temperature of $1.4 \mathrm{~K}$ was used. For the data presented below, an ac current $(500 \mathrm{nA}, 17.7 \mathrm{~Hz})$ is injected into the samples, and the longitudinal and Hall voltages are measured using lock-in amplifiers, which are attached to suitable voltage probes. Both cryostats are equipped with a rotatable sample stage, enabling scans of the angle $\Theta$ between the magnetic field direction and the plane of the 2DEG.

\section{MAGNETOTRANSPORT MEASUREMENTS}

In this section, we start by describing the magnetoresistivity of the 2DLGs with $n^{*}=0$, where only intrinsic obstacles are present and edge scattering is marginal, Figs. 1(a) and 1(b). We then look at the effects of artificial obstacles with successively increasing densities. Later on, further parameters are changed, namely the width of the Hall bar in Fig. 1(c) as well as the in-plane magnetic field in Fig. 2. Finally, the high-temperature regime $(\mathrm{T}>1 \mathrm{~K})$ is described in Fig. 3.

Figure 1 shows an overview of the magnetoresistivity as a function of $n^{\star}$, the temperature $T$ and $w$. For $w=100 \mu \mathrm{m}$ and in the absence of artificial scatterers $\left(n^{\star}=0\right)$, the typical GNMR structure is observed [lowermost two traces in
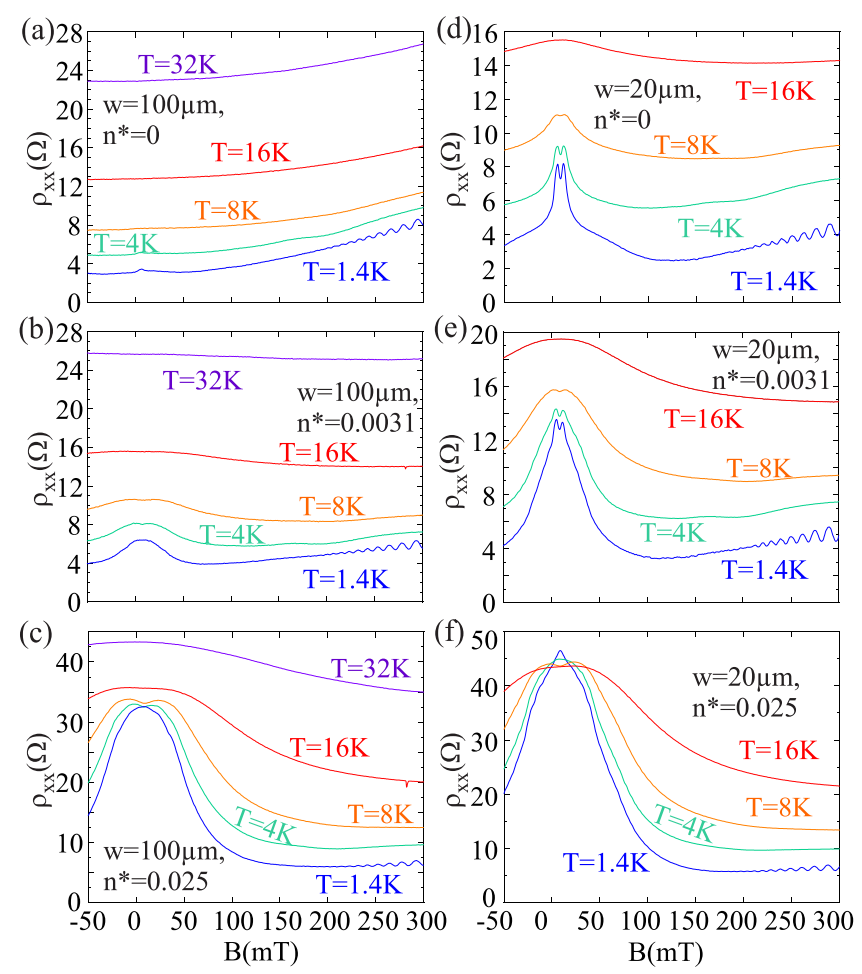

FIG. 3. Temperature dependence of the longitudinal magnetoresistivities at higher temperatures. Left column: $w=100 \mu \mathrm{m}$ with $n^{\star}=0$ (a), 0.0031 (b), and 0.025 (c). Right column: $w=20 \mu \mathrm{m}$ with $n^{\star}=0$ (d), 0.0031 (e), 0.025 (f).

Fig. 1(b)], very similar to the reports by Bockhorn et al. [3,5,30] as well as by Hatke et al. [6]: A narrow, approximately temperature-independent peak, centered at $B=0$ is located on top of a broad peak, which vanishes as the temperature is increased to $800 \mathrm{mK}$. As $n^{\star}$ is increased, the amplitude and the width of the temperature-independent part increase and the temperature-dependent component remains visible only at successively larger $B$ fields. In Fig. 1(c), the measurements on identical arrays in a Hall bar with $w=20 \mu \mathrm{m}$ are shown. For $n^{\star}=0$, a temperature-independent peak is centered at $B \approx 3 \mathrm{mT}$. The broad, temperature-dependent part extends to $B \approx 150 \mathrm{mT}$ and is thus significantly broader as compared to the $w=100 \mu \mathrm{m}$ Hall bar. Also, in the narrow Hall bar, $\rho_{x x}$ has increased significantly over the whole interval in which the GNMR structure is visible, indicating a nonlocal contribution to the resistivity. The temperature dependence sets in at similar magnetic fields as compared to Fig. 1(b), but it is weaker.

The short, vertical bars in Figs. 1(b) and 1(c) indicate the 2DLG percolation thresholds. Remarkably, the onset of the temperature dependence occurs at a magnetic field roughly equal to this phase boundary. Increasing $n^{\star}$ in these samples suppresses the narrow peak at finite $\mathrm{B}$, and for $n^{\star}=0.025$, the GNMR peak shape and its temperature dependence is approximately independent of $w$. Furthermore, the insets in Figs. 1(b) and 1(c) exemplify some temperature dependencies very close to $B=0$. A minute decrease of $\rho_{x x}$ very close to $B=0$ for the arrays with $n^{\star}=0.025$ is observed as T increases. 
In Fig. 1(d), the magnetoresistivities for arrays with large $n^{\star}$ are reproduced. Here, $w$ equals $100 \mu \mathrm{m}$. For $n^{\star} \geqslant 0.065$, only the tail of the GNMR peak at large magnetic fields in the quantum regime remains temperature-dependent, while an additional, narrow peak at $B=0$, already barely visible at $n^{\star}=0.025$, emerges and becomes more pronounced as $n^{\star}$ increases to 0.26 , which is already close to the upper localization threshold [28] of the Lorentz gases $\left(n_{u}^{\star}=0.359\right)$.

Besides the dependence on the temperature and the sample width, a further characteristic property of the GNMR is its remarkable evolution as a function of in-plane magnetic fields $[5,6]$. Hitherto, this dependence has been studied by measuring the longitudinal magnetoresistance in tilted $B$-fields, i.e., in a simultaneously varying in-plane component $B_{\|}[5,6]$. As illustrated in the supplement, our samples show the same phenomenology in this kind of experiment, namely a suppression of the T-dependent component of the GNMR by $B_{\|}$, while the narrow, temperature-independent structure remains essentially unchanged [23]. For the analysis to follow, however, it strikes us as more expedient to study the evolution of $\rho_{x x}(B)$ with $B_{\|}$as an independent parameter. These measurements are implemented by rotating the sample by small angles $\Theta$ about the parallel orientation where the $2 \mathrm{DEG}$ lies in the plane of the magnetic field, i.e., $\Theta=0$. Since for small angles, $B_{\perp} \equiv B=$ $B_{\text {tot }} \sin (\Theta) \approx B_{\text {tot }} \Theta$ and $B_{\|}=B_{\text {tot }} \cos (\Theta) \approx B_{\text {tot }}$, the in-plane magnetic field can be considered as approximately constant in the range of angles used. For example, in a total magnetic field of $10 \mathrm{~T}$, a perpendicular magnetic field of $300 \mathrm{mT}$ corresponds to a rotation angle of 1.7 degrees. At this angle, $B_{\|}=9.995 \mathrm{~T}$.

For $n^{\star}=0$, the negative magnetoresistivity in the temperature-dependent regime is suppressed as $B_{\|}$is increased, leading to a positive magnetoresistance at $B_{\|}=12 \mathrm{~T}$, see Fig. 2(a). A similar behavior was observed in studies focusing on the influence of $B_{\|}$on microwave-induced resistance oscillations [31], which has been discussed later on [15]. The shape of the narrow, temperature-independent peak is hardly influenced by small values of $B_{\|}$. For $B_{\|}=12 \mathrm{~T}$, a dip is observed in $\rho_{x x}(B)$ at $B=0$, which we interpret in terms of the well-known parabolic positive magnetoresistivity of two-subband systems [32], generated here by the spin splitting. The additional modulation of the Shubnikov-de Haas oscillations at large magnetic fields is tentatively attributed to an increasing effective mass by $B_{\|}$[33], although a detailed analysis is beyond our scope here. Furthermore, $\rho_{x x}\left(B_{\|}\right)$for $B=0$ increases approximately parabolically [Fig. 2(b)], by a factor reaching 1.43 at $B_{\|}=12 \mathrm{~T}$. The effect of a $2 \mathrm{DLG}$ on this behavior is exemplified for the array with $n^{\star}=0.0031$, see Fig. 2(c). Also here, the temperature-independent part of the GNMR is influenced only weakly by parallel magnetic fields up to $\approx 6 \mathrm{~T}$, and shows signatures of spin splitting at large $B_{\|}$. The data of further arrays are given within the Supplemental Material [23]. In the temperature-dependent interval of the GNMR, an increase of $\rho_{x x}$ comparable to that one for $n^{\star}=0$ is observed. We can thus conclude that moderate in-plane magnetic fields increase the scattering rates, which lead to an increase of the resistivities, but changes of the shape of $\rho_{x x}(B)$ are mostly observed in its temperature-dependent interval. Qualitatively, this behavior is independent of the Hall bar width (see Supplemental Material [23]). The quantum scattering time $\tau_{q}$ can be extracted from the envelope of the
Shubnikov-de Haas oscillations [34]. It tends to decrease with increasing $B_{\|}$as well as with increasing $n^{\star}$, as shown in Fig. 2(d). It is interesting to note that the effect of increasing the parallel magnetic field by $3 \mathrm{~T}$ has an approximately similar effect on $\tau_{q}$ as an increase of the obstacle density by a factor of four.

In Fig. 3, some typical magnetoresistivities at higher temperatures, $T>1 \mathrm{~K}$, are shown. For our larger Hall bar width $(w=100 \mu \mathrm{m})$ without artificial obstacles, the GNMR structure that has vanished around $800 \mathrm{mK}$ remains absent and a broad, positive magnetoresistivity is formed, see Fig. 3(a). For $T=4 \mathrm{~K}$ and $8 \mathrm{~K}$, a weak modulation is superimposed, which we attribute to phonon-induced resistance oscillations (PIRO) [20,35]. With increasing values of $n^{\star}$, the GNMR peak becomes more robust with respect to thermal smearing and is still visible at $T=16 \mathrm{~K}$ [Figs. 3(b) and 3(c)]. Also here, PIRO are visible at intermediate temperatures, with an additional peak appearing at smaller magnetic fields around [9]. A smaller Hall bar width $(w=20 \mu \mathrm{m}$, right column of Fig. 3) stabilizes the GNMR structure with respect to thermal activation. Most dramatically, the peak observed in the sample with $n^{\star}=0$ remains visible up to $T=16 \mathrm{~K}$, and the PIRO are quite pronounced. As in our wide Hall bar, increasing $n^{\star}$ makes the magnetoresistance peaks even more robust with respect to an increasing temperature, see Figs. 3(e) and 3(f).

\section{INTERPRETATION AND DISCUSSION}

In this section, the various aspects of the GNMR as specified experimentally above will be analyzed and discussed in greater detail.

\section{A. The transition magnetic field}

The observations reported above suggest that the component of the GNMR with a weak dependence on temperature and in-plane magnetic field is dominated by strong scattering with a classical character. The shape of this component depends on the obstacles that contribute to the elastic scattering rate, namely the Lorentz array, the sample edges as well as some unknown potential landscape of the pristine 2DEG. Above a threshold magnetic field, the temperature-dependent component, which also depends strongly on $B_{\|}$becomes dominant. The values of this threshold are found to be very similar to $B_{c}\left(n^{\star}\right)$, the lower percolation threshold of the 2DLG. This suggests the following picture, see also Fig. 1(a). At magnetic fields below $B_{c}$, electrons are delocalized, and the resistivity is dominated by the strong, elastic scattering at the obstacles. Therefore, it has a weak $\mathrm{T}$ dependence. Perfect agreement with the theoretical value for $B_{c}$ is not to be expected, considering that it disregards scattering at the edges and at further scatterers in the bulk. In a clean 2DLG above $B_{c}$, all electrons are localized in skipping orbits around the obstacles or clusters thereof, as well as in closed, undisturbed cyclotron orbits. In this phase, the transport in a real system has an activated character, since additional background scattering, for example by phonons, disturbs the cyclotron motion and tends to unpin the electrons from the obstacles [22,28,36,37]. In fact, the magnetoconductance in this regime increases as the temperature is increased, which becomes apparent by transforming the data 
of Figs. 1(a) and 1(b) to longitudinal magnetoconductivities $\sigma_{x x}(B)=\rho_{x x}(B) /\left[\rho_{x x}^{2}(B)+\rho_{x y}^{2}(B)\right]$, shown in Fig. 5 within the Supplemental Material [23]. From this viewpoint, it appears plausible to relate the value for $B \approx 16 \mathrm{mT}$ at which the temperature dependence sets in for the sample with $n^{\star}=0$, Fig. 1(b), to an effective dimensionless obstacle density of $n_{2 \mathrm{DEG}}^{\star} \approx 0.002$. Thus, strong scatterers like, e.g., oval defects, of the size of one obstacle from our Lorentz gases would have an average distance of $\approx 11 \mu \mathrm{m}$, with a plausible mean free path of $\ell_{s} \approx 125 \mu \mathrm{m}$. It should be noted that the interpretation of the transition magnetic field provided by Bockhorn et al. in terms of scattering at oval defects $[3,5,30]$ is in full agreement with the conclusions drawn here.

\section{B. Shape of the temperature-independent component}

We proceed with a closer look at the narrow, temperatureindependent magnetoresistance structures close to $B=0$. A peak at $B=0$ is visible in the Hall bars of $100 \mu \mathrm{m}$ width for $n^{\star}=0$, which broadens as $n^{\star}$ is increased, while the samples with $w=20 \mu \mathrm{m}$ show a peak at $B=3 \mathrm{mT} n^{\star}=0$, which sits on top of a broader, T-independent shoulder and which is suppressed for increasing $n^{\star}$.

\section{Dependence on $n^{\star}$ and $w$; the mixed disorder model}

We interpret the peak at $B>0$ as a signature of diffusive scattering at the edges of the Hall bar. It is well known that this type of edge scattering causes a magnetoresistance peak centered at $w \approx 0.55 r_{c}$ [38] where $r_{c}$ denotes the cyclotron radius, i.e., at a magnetic field $B_{w p} \approx(0.55 / w) \times(\hbar / e) \times$ $\sqrt{2 \pi n}$, leading to expected peak positions of $B_{w p}(w=$ $20 \mu \mathrm{m})=2.3 \mathrm{mT}$ and $B_{w p}(w=100 \mu \mathrm{m})=0.45 \mathrm{mT}$. Since the shape of this peak is not well understood but it is known from experiments to have a pronounced tail towards larger magnetic fields [38], boundary scattering may contribute significantly to the narrow magnetoresistance structure even for wider Hall bars where the position of the wire peak cannot be distinguished from $B=0$. Thus, diffusive boundary scattering may contribute, for example, to the approximately constant $\rho_{x x}(B)$ close to $B=0$ in the samples with $n^{\star}=0.0031$ and 0.0062 with $W=100 \mu \mathrm{m}$, see Fig. 1(b). As $n^{\star}$ increases, edge scattering becomes less relevant, and the wire peak tends to vanish for $n_{s}^{-1 / 2} \ll w$. This is in good agreement with our experimental observations. Therefore, it appears plausible that the dependence of this part of the GNMR on $w$ may be due to diffusive edge scattering. In experiments where the shape of this structure is independent of $w$ [5], $n_{s}^{-1 / 2}$ may be comparable to or smaller than $w$, or the walls may have a high specularity. However, just the absence of a classical resistivity peak at nonzero $B$ is not sufficient to exclude an edge scattering contribution.

A quantitative analysis of this temperature-independent GNMR peak component at $B=0$ in terms of mixed disorder scattering $[18,36]$ has been carried out by Bockhorn et al. [5] as well as by Hatke et al. [6]. Within this model, the negative magnetoresistance for $B$ below the percolation threshold originates from the skipping trajectories of the electrons around and in between strong scatterers (here predominantly the Lorentz obstacles), which are disturbed by the weak background scattering. For infinite samples (disregarding edge
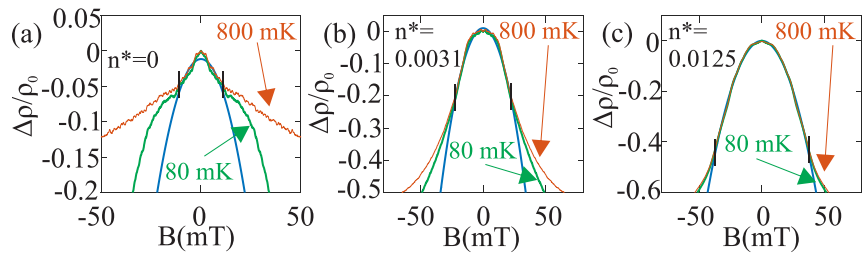

FIG. 4. Fits (blue lines) of the narrow peaks of $\rho_{x x}(B)$ at $80 \mathrm{mK}$ (green lines) to Eq. (2) for $n^{\star}=0$ (a), 0.0031 (b), and 0.0125 (c). The corresponding fit parameters for density of strong scatterers are $n_{s, \text { eff }}=2.80 \times 10^{8} \mathrm{~m}^{-2}(\mathrm{a}), n_{s, \text { eff }}=1.68 \times 10^{9} \mathrm{~m}^{-2}(\mathrm{~b})$, and $n_{s, \text { eff }}=$ $1.79 \times 10^{10} \mathrm{~m}^{-2}$ (c). For comparison, the traces at $800 \mathrm{mK}$ are shown as well (orange lines). The black vertical bars denote the limits of the fit interval, and $n_{s}$ is the fit parameter value obtained.

scattering) and for scattering times of the weak background disorder $\tau_{L}$ much larger than $\tau_{S}$, the scattering time by strong disorder, the peak can be modeled by

$$
\rho_{x x}(B)=\rho_{x x}(0)\left[1-\frac{\omega_{c}^{2} f\left(\tau_{S} / \tau_{L}\right)}{2 \pi n_{s} v_{F}^{2}}\right],
$$

with the cyclotron frequency $\omega_{c}$ and the function

$$
f(x) \equiv \frac{2}{x+1} \int_{0}^{\infty} \frac{y J_{1}^{2}(y)}{x y^{2}+2\left(1-J_{0}^{2}(y)\right)} d y,
$$

where $J_{i}(y)$ denote Bessel functions in the conventional notation [5].

Here, $\tau_{L} \approx 320 \mathrm{ps}$ is estimated from $\rho_{x x}\left(B_{c}\right)=\frac{m^{*}}{n_{e} e^{2} \tau_{L}}[5]$ of the $n^{\star}=0$ sample and it is assumed that the Matthiesen rule holds for the combined scattering, i.e., $\rho_{x x}(0)=\frac{m^{\star}}{n e^{2}}\left(\tau_{S}^{-1}+\right.$ $\tau_{L}^{-1}$ ), which gives the corresponding value for $\tau_{S}$ from the measurements, but is strictly valid only for sufficiently small $n^{\star}$. Thus, fits of Eq. (2) to the measurements of $\rho_{x x}(B)$ in the interval $B \leqslant B_{c}$ with the effective density of strong scatterers $n_{s}^{\text {eff }}$ as fit parameter can be carried out. The results are shown in Fig. 4 for three exemplifying values of $n^{\star}$.

For $n^{\star}=0, \rho_{x x}(B)$ can hardly be called parabolic, and the fit is accordingly quite poor. The obtained fit value of $n_{s, \text { eff }}=2.8 \times 10^{8} \mathrm{~m}^{-2}$ corresponds to quite large average distance between strong scatterers of $60 \mu \mathrm{m}$. Comparable results have been reported earlier [5], where, however, an influence of edge scattering could be excluded. As $n^{\star}$ is increased, the parabolicity of the magnetoresistance improves. The fit values are smaller than the designed obstacle densities but approach them as $n^{\star}$ increases. In particular, they are a factor of 7.4 (2.8) smaller than the lithographic obstacle densities at $n^{\star}=0.0031(0.0125)$. For $n^{\star}=0.025$, the ratio drops to 1.68 (the fit value was $n_{s, \text { eff }}=5.95 \times 10^{10} \mathrm{~m}^{-2}$ ), thereby leading to a rather accurate estimate of the average obstacle spacing with a deviation of $30 \%$. We attribute this to the fact that the condition $\tau_{L} \gg \tau_{S}$ is only fulfilled for sufficiently large $n^{\star}$, i.e., $\tau_{L} / \tau_{S}=0.063$ for $n^{\star}=0$ and 9.1 for $n^{\star}=0.025$. Thus, the samples showing a typical GNMR structure [5,6,9] are most likely outside the range of validity of the mixed disorder model, and fitting the GNMR data to this model in the regime $\tau_{L} \leqslant \tau_{S}$ underestimates the density of the strong scatterers. For example, samples A and B in Ref. [6] resemble our samples with $n^{\star}=0.0062$ and 0 , respectively, and the authors 
have estimated $\tau_{L} \approx 0.2 \tau_{S}$ for their sample $\mathrm{A}$. The samples studied by Shi et al. [9], appear to have a rather large density of strong scatterers with an effective $n^{\star} \approx 0.01$, but the mixed disorder model predicts a much broader magnetoresistivity peak. This indicates that the ratio $\tau_{L} / \tau_{S}$ is still too small for the applicability of the mixed disorder model. Furthermore, our study indicates that contributions from edge scattering may influence this part of the GNMR peak even if a wire peak is not visible. The studies reported by Mani et al. on samples of very high mobility show a characteristic narrow peak at $B=0$ on top of a broad one, a structure, which resembles our data for samples with $n^{\star}=0$ [7]. The narrow peak observed in this experiment has been interpreted in terms of weak localization, however. We can exclude this explanation for our samples, since the magnetoconductivity is negative for all values of $n^{\star}$ (see Supplemental Material [23]), while a positive magnetoconductivity is expected for weak localization, due to the suppression of coherent backscattering in magnetic fields [39].

Interestingly, as $n^{\star}$ is increased into the regime where kinetic models are inapplicable [22] $\left(n^{\star} \gtrsim 0.025\right), \rho_{x x}(B)$ develops a structure, which resembles the GNMR, namely a narrow peak at $B=0$ on top of a much broader shoulder. However, both features are robust with respect to temperatures and in-plane magnetic fields. They have been explained in terms of classical memory effects $[17,18,22,26,27]$, where the broad peak corresponds to the narrow peak in the GNMR regime, and the additional structure on top is attributed to retroreflection at the Lorentz obstacles, with an approximately linear negative magnetoresistance $[19,25]$. This shows that a classification of the GNMR based on just the shape at a fixed temperature is insufficient.

\section{The weak dependence on $B_{\|}$}

In Fig. 2(d), it has been shown that $\tau_{q}$ is strongly influenced by $B_{\|}$: It decreases by a factor of 3 as $B_{\|}$is increased from 0 to $3 \mathrm{~T}$. As elaborated in Ref. [40], the position of the electronic wave function in growth direction of the heterostructure depends on the in-plane magnetic field, and the direction-averaged transport scattering time, which no longer depends in a simple way on the quantum scattering time, decreases due to the scattering contributions of the wave vectors for which the wave function is displaced towards the remote donors. This causes a decrease of the scattering times and a positive magnetoresistance as a function of both the perpendicular as well as the in-plane magnetic field, as observed here as well, see Figs. 2(a) and 2(c). Therefore, we attribute the weakly increasing resistivity by $B_{\|}$close to $B=0$ in our samples to this established effect. However, the shape of the temperature-independent GNMR component changes only marginally, indicating that the additional scattering is essentially irrelevant for the unpinning of the electrons from the obstacles. This can be understood with the picture of the two phases of the 2DLG: here, the system is still in the conducting phase, where delocalized electrons move in straight or weakly bent trajectories through the sample, a motion, which experiences only minor changes by the additional weak scattering.
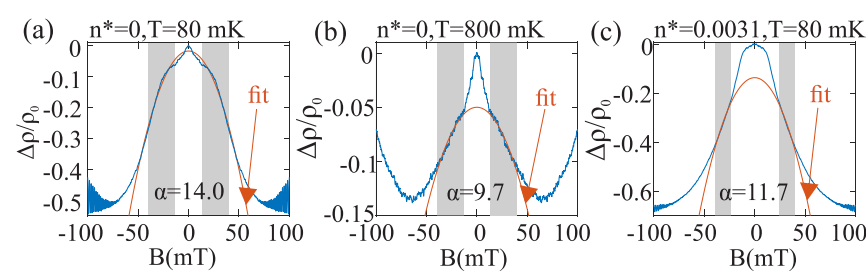

FIG. 5. Parabolic fits of the temperature-dependent part of the GNMR for the pristine sample $\left(n^{\star}=0\right)$ at base temperature (a) and for $800 \mathrm{mK}$ (b), using $\alpha$ [see Eq. (3)] as fit parameter. The corresponding fit for $n^{\star}=0.0031$ at base temperature is shown in (c) The gray regions denote the fit intervals, with $B_{t}$ as the lower and $40 \mathrm{mT}$ as the upper boundary, respectively. All measurements here have been taken on the samples with $w=100 \mu \mathrm{m}$.

\section{Properties of the temperature-dependent component}

As for the discussion of the temperature-independent part above, we structure this subsection according to the different aspects of this component.

\section{The interaction-induced resistivity correction}

The broad, temperature-dependent part of the GNMR has been analyzed by Hatke et al. [6] as well as by Bockhorn et al. [4] within the model of an interaction-induced correction in the presence of long- and short-range disorder [41]. This model predicts a parabolic, temperature-dependent magnetoresistance given by

$$
\frac{\rho_{x x}(B)}{\rho(0)}=1-\frac{\alpha c_{0}}{2 n^{2} \pi^{2} \sqrt{\hbar k_{B} T \tau}},
$$

where $c_{0} \approx 0.276$ is a numerical constant and $\alpha$ is a parameter that depends on the ratio of the Drude to the quantum scattering time, i.e., $\alpha=4 \sqrt{\tau / \tau_{q}} /\left(k_{F} d\right)$ with the Fermi wave vector $k_{F}$ and the distance $d$ between the 2DEG and the donor layer, which in our heterostructure equals $d=40 \mathrm{~nm}$.

As in the studies reported earlier $[4,6]$, we do not observe the predicted $T^{-0.5}$ scaling, but rather a dependence roughly $\propto 1 / T$ (not shown). For our sample with $n^{\star}=0$ where $\tau=$ $290 \mathrm{ps}$ and $\tau_{q} \approx 3.5 \mathrm{ps}$ (see Supplemental Material [23]), we expect $\alpha=7.2$. In analogy to the analysis provided in earlier work $[4,6]$, we compare this value to those obtained by fitting Eq. (3) to our experimental data of the samples with $w=100 \mu \mathrm{m}$ using $\alpha$ as fit parameter and obtain values $\alpha_{\mathrm{fit}}$ for different temperatures, which deviate from 7.2 by a factor between 1.95 at base temperature and 1.35 at $800 \mathrm{mK}$, where, however, the measured trace is only approximately parabolic at best, see Figs. 5(a) and 5(b).

As $n^{\star}$ is increased, a magnetic field interval in which the temperature dependent part is well described by a parabola can no longer be identified, see Fig. 5(c). Possibly, contributions from the strong scatterers as well as the larger relevance of the $E \times B$ drift at the sample edges influence the magnetoresistance in this regime. A further reason for the nonparabolicity may be that even for our lowest nonvanishing value of $n^{\star}=0.0031$, the obstacle density is too large for Eq. (3) to be strictly valid. Nevertheless, a fit of $\rho_{x x}(B)$ in this regime to Eq. (3) gives quite comparable values for $\alpha$ to those found for $n^{\star}=0$. 


\section{The dependence on parallel magnetic fields}

As shown in Figs. 2(a) and 2(b), and in agreement with earlier reports $[5,6]$, the temperature-dependent part of $\rho_{x x}(B)$ broadens and changes its shape under the influence of inplane magnetic fields. To the best of our knowledge, there is no model available that extends the interaction effects in the presence of mixed disorder to $B_{\|}$. Qualitatively, however, it appears plausible that the broadening of the GNMR by in-plane magnetic fields can be caused by the decrease of the quantum scattering time: the system is in the localized phase, where transport requires un-pinning of the electrons from the Lorentz obstacles by additional scattering, such as that one indicated by a decreasing $\tau_{q}$. We note that Zeeman splitting may contribute to the non-monotonic shape of $\rho_{x x}(B)$ as well.

\section{The dependence on the sample width $w$}

Furthermore, the broad, temperature-dependent peak has been reported to depend on $w$, scaling roughly with $w^{-0.5}$ [7]. In our experiments, the scaling factor deceases monotonically as $n^{\star}$ is increased, from 1.9 for $n^{\star}=0$ to 1.15 for $n^{\star}=0025$ (Fig. 2 within the Supplemental Material [23]). This suggests that the scaling factor is a measure for the significance of edge scattering, approaching 1 as the distance between the Lorentz obstacles gets much smaller than w. We have determined the quantum scattering time for the Hall bars of $20 \mu \mathrm{m}$ to $\tau_{q}=$ $3.5 \mathrm{ps}$ for $n^{\star}=0$, quite similar to that one for the samples with $100 \mu \mathrm{m}$, indicating that weak scattering at the edges is marginal. Therefore, we tentatively explain the dependence of this part of the GNMR on $w$ by strong scattering at the edges, similar in character to the scattering at the Lorentz obstacles.

\section{The high-temperature regime}

We proceed with a discussion of $\rho_{x x}(B)$ at higher temperatures, i.e., in the range $T \epsilon[1.4 \mathrm{~K}, 32 \mathrm{~K}]$ shown in Fig. 3. Since the component of the GNMR with a significant temperature dependence below $1 \mathrm{~K}$ has decayed in this regime, we observe here the thermal smearing of the GNMR component we called temperature-independent in the measurements below $1 \mathrm{~K}$. Such a weak temperature dependence is in tune with our earlier identification of this component as classical, predominantly elastic scattering. Apparently, a decrease of the mean free path by Lorentz circles or at sample edges, increases the pronunciation and the thermal stability of the peak, with a remarkably strong influence of edge scattering in the samples with the narrow Hall bar. A higher temperature increases the electron-phonon scattering rate and changes thereby the ratio $\tau_{L} / \tau_{S}$ towards smaller values, while the character of the strong scattering remains nonrandom. In this regime, therefore, all samples are outside the range of validity of the mixed disorder model, and a corresponding theoretical description is not available to the best of our knowledge. The magnetoresistivities reported by Shi et al. [9], as well as those shown by Hatke et al. [6] for their sample A, somewhat resemble our data shown in Fig. 3(c). Hence, possibly, also in those samples, $\rho_{x x}(B)$ may be governed by this regime of scattering times. The data of Shi et al. have been interpreted later on in terms of a hydrodynamic model [12], where the electron-electron scattering rate increases with $T$, thereby decreasing the viscosity of the electron liquid. Since the viscosity is suppressed by perpendicular magnetic fields, a negative magnetoresistance is expected. Within this model, the resistivity is determined by a Hagen-Poiseuille-type flow profile with zero velocity at the edges of the strong scatterers. Hence, the Lorentz obstacles determine, together with the sample edges, an effective width $w_{\text {eff }}$ in between which the flow profile is established [12]. The hydrodynamic model predicts a decreasing resistivity as the temperature is increased, due to the decreasing viscosity, as has been observed by Levin et al. [42] in 2DEGS of much larger electron densities and in wires of $5 \mu \mathrm{m}$ and below. We observe a resistivity, which increases with $T$ at $B=0$ and develops a positive dependence on $\mathrm{B}$ at large temperature. This suggests that the hydrodynamic model is inadequate for an explanation of our data. Nevertheless, we have tentatively fitted some of our measurements to the hydrodynamic model, with the relevant time scales as fit parameters (see Supplemental Material [23]). For example, for the sample with $n^{\star}=0.062$ and $w=100 \mu \mathrm{m}$, a fit value for the Drude scattering time of $426 \mathrm{ps}$ at $T=1.4 \mathrm{~K}$ is obtained, which is roughly a factor of 5 larger than the value obtained from the resistivity at $B=0$. While our observations in the high temperature regime are consistent with an interpretation in terms of predominantly classical scattering within a single particle picture including memory effects, we do not exclude the possibility that in some parameter range, in particular at high temperatures, larger electron densities and/or small Hall bar widths, a hydrodynamic description may be appropriate, as has been shown recently for some other systems in this temperature regime [43-45]

\section{SUMMARY AND OUTLOOK}

We have presented a study of the GNMR structure on a series of samples where strong (via the density of Lorentz obstacles and the sample width) and weak (via temperature and in-plane magnetic fields) scattering is varied. The GNMR structure shows a continuous, multicomponent evolution as a function of the corresponding scattering times. As the density of strong obstacles increases, the temperatureindependent component of the GNMR close to $B=0$ extends over successively larger intervals, with the transition to the temperature-dependent component taking place close to the percolation threshold of the Lorentz gas. Thus, the behavior in this phase is based on the transport properties of classically delocalized states. For low obstacle densities, the existing models for mixed disorder potentials predict a parabolic shape for the two GNMR components. The curvature of the temperature-independent peak can be used to determine the density of the sparse, strong obstacles, and our study suggests that this fit can be used for an order-of-magnitude estimate of $n_{s}$. One source of error is edge scattering, which can disturb the parabolicity of the peak significantly. Furthermore, most samples studied so far have parameters outside the range of validity of the mixed disorder model, and our study suggests that in cases where the long-range scattering time is not large compared to the short-range scattering time, this analysis underestimates the density of strong scatterers. In the range of large obstacle densities where $n_{s}^{-0.5} \ll \ell_{e}$, a peak shape quite similar to the GNMR emerges, which is however quite 
robust with respect to thermal activation. In this regime, the narrow peak close to $B=0$ originates from retroreflection. A peak at nonzero magnetic field at low temperature can be attributed to edge scattering, while at higher temperatures, a quite similar structure can be caused by phonon-induced resistance oscillations [see in particular Figs. 3(c) and 3(d)].

The temperature-dependent part of the GNMR depends sensitively on the Drude and quantum scattering times. The system is in the classically localized phase, where additional weak scattering contributes to the unpinning of electrons that are trapped at, or in between, strong obstacles in the absence of weak disorder. Both scattering times are found to be decreased by edge scattering as well as by additional in-plane magnetic fields, which explains qualitatively the dependence of the GNMR width on these parameters. For a better quantitative agreement, the existing models should be extended to the regime where the scattering time for long range scattering is comparable to, or smaller than, the short range scattering time, and include further scattering contributions like edge scattering. Also, it would be helpful to look at interaction corrections in the presence of in-plane magnetic fields. In the future, it will be furthermore interesting to study the crossover from the mixed disorder model, for example in form of a 2DLG, to the hydrodynamic model. Finally, further insight might be gained from current-voltage characteristics in combination with magnetic field sweeps in the nonlinear regime, along the lines of Ref. [46].

\section{ACKNOWLEDGMENTS}

This work was supported by the French RENATECH network. We thank J. Horbach, L. Bockhorn and R. Haug for stimulating discussions.
[1] Y. Dai, R. R. Du, L. N. Pfeiffer, and K. W. West, Phys. Rev. Lett. 105, 246802 (2010).

[2] A. T. Hatke, M. A. Zudov, L. N. Pfeiffer, and K. W. West, Phys. Rev. B 84, 121301(R) (2011).

[3] L. Bockhorn, P. Barthold, D. Schuh, W. Wegscheider, and R. J. Haug, Phys. Rev. B 83, 113301 (2011).

[4] L. Bockhorn, A. Hodei, D. Schuh, W. Wegscheider, and R. J. Haug, J. Phys. Conf. Ser. 456, 012003 (2013).

[5] L. Bockhorn, I. V. Gornyi, D. Schuh, C. Reichl, W. Wegscheider, and R. J. Haug, Phys. Rev. B 90, 165434 (2014).

[6] A. T. Hatke, M. A. Zudov, J. L. Reno, L. N. Pfeiffer, and K. W. West, Phys. Rev. B 85, 081304(R) (2012).

[7] R. G. Mani, A. Kriisa, and W. Wegscheider, Sci. Rep. 3, 02747 (2013).

[8] V. Umansky, R. de Picciotto, and M. Heiblum, Appl. Phys. Lett. 71, 683 (1997).

[9] Q. Shi, P. D. Martin, Q. A. Ebner, M. A. Zudov, L. N. Pfeiffer, and K. W. West, Phys. Rev. B 89, 201301(R) (2014).

[10] L. Li, Y. Y. Proskuryakov, A. K. Savchenko, E. H. Linfield, and D. A. Ritchie, Phys. Rev. Lett. 90, 076802 (2003).

[11] E. A. Galaktionov, A. K. Savchenko, and D. A. Ritchie, Phys. Status Solidi C 3, 304 (2006).

[12] P. S. Alekseev, Phys. Rev. Lett. 117, 166601 (2016).

[13] P. S. Alekseev and M. A. Semina, Phys. Rev. B 98, 165412 (2018).

[14] J. Iñarrea, Europhys. Lett. 106, 47005 (2014).

[15] A. Bogan, A. T. Hatke, S. A. Studenikin, A. Sachrajda, M. A. Zudov, L. N. Pfeiffer, and K. W. West, Phys. Rev B 86, 235305 (2012)

[16] H. A. Lorentz, Proc. R. Acad. Sci. Amsterdam 7, 438 (1905).

[17] A. D. Mirlin, D. G. Polyakov, F. Evers, and P. Wölfle, Phys. Rev. Lett. 87, 126805 (2001).

[18] D. G. Polyakov, F. Evers, A. D. Mirlin, and P. Wölfle, Phys. Rev. B 64, 205306 (2001).

[19] A. Dmitriev, M. Dyakonov, and R. Jullien, Phys. Rev. Lett. 89, 266804 (2002).

[20] I. A. Dmitriev, A. D. Mirlin, D. G. Polyakov, and M. A. Zudov, Rev. Mod. Phys. 84, 1709 (2012).
[21] Y. M. Beltukov and M. I. Dyakonov, Phys. Rev. Lett. 116, 176801 (2016).

[22] N. H. Siboni, J. Schluck, K. Pierz, H. W. Schumacher, D. Kazazis, J. Horbach, and T. Heinzel, Phys. Rev. Lett. 120, 056601 (2018).

[23] See Supplemental Material at http://link.aps.org/supplemental/ 10.1103/PhysRevB.104.045306 for complementary measurements for additional sample parameters.

[24] J. Ferenc and Z. Neda, Physica A 385, 518 (2007).

[25] J. Schluck, M. Hund, T. Heckenthaler, T. Heinzel, N. H. Siboni, J. Horbach, K. Pierz, H. W. Schumacher, D. Kazazis, U. Gennser, and D. Mailly, Phys. Rev. B 97, 115301 (2018).

[26] E. M. Baskin, L. N. Magarill, and M. V. Entin, Sov. Phys. JETP 48, 365 (1978).

[27] A. V. Bobylev, F. A. Maaø, A. Hansen, and E. H. Hauge, Phys. Rev. Lett. 75, 197 (1995).

[28] A. Kuzmany and H. Spohn, Phys. Rev. E 57, 5544 (1998).

[29] J. Schluck, S. Fasbender, T. Heinzel, K. Pierz, H. W. Schumacher, D. Kazazis, and U. Gennser, Phys. Rev. B 91, 195303 (2015).

[30] L. Bockhorn, A. Velieva, S. Hakim, T. Wagner, E. P. Rugeramigabo, D. Schuh, C. Reichl, W. Wegscheider, and R. J. Haug, Appl. Phys. Lett. 108, 092103 (2016).

[31] C. L. Yang, R. R. Du, L. N. Pfeiffer, and K. W. West, Phys. Rev. B 74, 045315 (2006).

[32] E. Zaremba, Phys. Rev. B 45, 14143 (1992).

[33] A. T. Hatke, M. A. Zudov, L. N. Pfeiffer, and K. W. West, Phys. Rev. B 85, 241305(R) (2012).

[34] T. Ando, J. Phys. Soc. Jpn. 37, 1044 (1974).

[35] M. A. Zudov, I. V. Ponomarev, A. L. Efros, R. R. Du, J. A. Simmons, and J. L. Reno, Phys. Rev. Lett. 86, 3614 (2001).

[36] A. D. Mirlin, E. Tsitsishvili, and P. Wölfle, Phys. Rev. B 63, 245310 (2001).

[37] B. Horn-Cosfeld, J. Schluck, A. Röben, M. Cerchez, K. Pierz, H. W. Schumacher, D. Mailly, and T. Heinzel, EPL 128, 67004 (2019). 
[38] T. J. Thornton, M. L. Roukes, A. Scherer, and B. P. Van de Gaag, Phys. Rev. Lett. 63, 2128 (1989).

[39] S. Hikami, A. I. Larkin, and Y. Nagaoka, Prog. Theor. Phys. 63, 707 (1980).

[40] J. M. Heisz and E. Zaremba, Phys. Rev. B 53, 13594 (1996).

[41] I. V. Gornyi and A. D. Mirlin, Phys. Rev. B 69, 045313 (2004).

[42] A. D. Levin, G. M. Gusev, E. V. Levinson, Z. D. Kvon, and A. K. Bakarov, Phys. Rev. B 97, 245308 (2018).
[43] F. M. D. Pellegrino, I. Torre, and M. Polini, Phys. Rev. B 96, 195401 (2017).

[44] T. Scaffidi, N. Nandi, B. Schmidt, A. P. Mackenzie, and J. E. Moore, Phys. Rev. Lett. 118, 226601 (2017).

[45] L. V. Delacrétaz and A. Gromov, Phys. Rev. Lett. 119, 226602 (2017).

[46] Q. Shi, M. A. Zudov, L. N. Pfeiffer, and K. W. West, Phys. Rev. B 90, 201301(R) (2014). 